\title{
PBTL CD19CAR-28/CD137/zeta
}

National Cancer Institute

\section{Source}

National Cancer Institute. PBTL CD19CAR-28/CD137/zeta. NCI Thesaurus. Code C106429.

Peripheral blood T-lymphocytes (PBT Ls) transduced with a retroviral vector expressing a chimeric antigen receptor (CAR) consisting of an anti-CD19 scFv (single chain variable fragment) coupled to the costimulatory signaling domain CD28, the signaling domain of 4-1BB (CD137), and the zeta chain of the T-cell receptor (TCR), with potential immunomodulating and antineoplastic activities. Upon transfusion, PBTL CD19CAR28/CD137zeta directs the T-lymphocytes to CD19-expressing tumor cells and induces selective toxicity in CD19-expressing tumor cells. CD28, a T-cell surface-associated costimulatory molecule, is required for full T-cell activation, proliferation, and survival. The 4-1BB co-stimulatory molecule signaling domain enhances activation and signaling after recognition of CD19. Furthermore, inclusion of the 4-1BB signaling domain may increase the antitumor activity compared to the inclusion of the CD28 costimulatory domain and TCR zeta chain alone. CD19 antigen is a B-cell specific cell surface antigen, which is expressed in all B-cell lineage malignancies. 